\title{
Simulation of Ion Motion at Atmospheric Pressure: Particle Tracing Versus Electrokinetic Flow
}

\author{
Walter Wissdorf, ${ }^{1}$ Larissa Pohler, ${ }^{2}$ Sonja Klee, ${ }^{1}$ David Müller, ${ }^{1}$ Thorsten Benter ${ }^{1}$ \\ ${ }^{1}$ Department of Physical and Theoretical Chemistry, University of Wuppertal, Gauss Str. 20, 42119 Wuppertal, Germany \\ ${ }^{2}$ Department of Chemistry, University of Duesseldorf, Duesseldorf, Germany
}

\begin{abstract}
Results obtained with two computational approaches for the simulation of ion motion at elevated pressure are compared with experimentally derived ion current data. The computational approaches used are charged particle tracings with the software package SIMION ver. 8 and finite element based calculations using the software package Comsol Multiphysics ver. 4.0/4.0a. The experimental setup consisted of a tubular corona discharge ion source coupled to a cylindrical measurement chamber held at atmospheric pressure. Generated ions are flown into the chamber at essentially subsonic laminar isothermal conditions. In the simulations, strictly stationary conditions were assumed. The results show very good agreement between the SIMION/SDS model and experimental data. For the Comsol model, only qualitative agreement is observed.
\end{abstract}

Key words: Simulation, SIMION, FEM, Comsol, CFD, Particle tracing, Electro dynamics

\section{Introduction}

$\mathrm{T}$ he design and optimization of ion optical devices is a common task during the development stage of, e.g., ion optical elements, mass spectrometers (MS), or ion mobility spectrometers (IMS). The numerical simulation of the motion of charged particles within corresponding force fields is an extremely useful tool, which drastically reduces the cost, time, and effort needed for the design process. Reliable computer models based on validated experimental data allow the in-depth investigation of the characteristics of ion optical designs without the need of physical fabrication.

Within high vacuum environments and, thus, at virtually collision-free conditions, ion motion is solely governed by the presence of electric and magnetic fields. Over the past three decades, the simulation of the motion of ions in collision-free environments with software packages (e.g., SIMION [1]) has matured to a standard method in the industrial and scientific community. Today, even highly

Correspondence to: Walter Wissdorf; e-mail: wissdorf@uni-wuppertal.de, Thorsten Benter; e-mail: tbenter@uni-wuppertal.de complex models are simulated and analyzed on state-of-theart generic consumer computer hardware.

The introduction and rapid evolution of novel atmospheric pressure ionization methods, e.g., the emerging methods based on electrospray ionization (ESI) [2] or atmospheric pressure chemical ionization (APCI) [3], and new ion analyzer concepts, e.g., the combination of IMS and MS [4, 5], lead to a steeply rising demand for ion motion simulations at elevated or even atmospheric pressure (AP).

At atmospheric pressure, each ion experiences about $10^{9}$ collisions per s with bulk gas ("matrix") molecules [6], i.e., there are extensive interactions with the surrounding gas. Consequently, the dynamics of the neutral bulk gas as well as ion diffusion have to be considered in elevated pressure ion motion models. There are at least two established numerical approaches for the simulation of ion motion at elevated pressure.

The first approach is based on discrete particle motion. The widely applied program package, SIMION, offers a user program interface, which allows the direct interaction with the simulation algorithm for charged particle trajectory calculations. In 2005, Dahl and coworkers [7] introduced 
an extension to SIMION based on this user program interface, which implements high pressure ion motion simulation in terms of a statistical diffusion simulation (SDS) algorithm.

Alternatively, the direct handling of individual collision interactions between ions and bulk gas molecules have been described [8]. At atmospheric pressure, however, the number of collisions per simulation step increases to an extent that currently renders the simulation numerically too expensive.

The SDS algorithm [7] does not take into account individual collisions; rather, the ion motion is described by a viscous drift and an independent diffusion motion. The viscous interaction with the bulk gas is treated with a Stokes-Law model. The ion diffusion is modeled in terms of single random "jumps" for each simulation time step, which represents the net effect of the many collisions that occurred in the time interval between the simulation steps. The statistical parameters of the random jumps were established while developing the SDS algorithm. The details of the SDS process along with the underlying collision statistical investigations are available in the literature [7].

The second approach describes the ion motion as an evolving concentration distribution in a continuous model, rather than as discrete moving particles. Here, the migration of the ions within the electrical field and their convective and diffusional transport is described by the transport Equation (1):

$$
\frac{d c}{d t}+\nabla(-D \nabla c-z K F c \nabla V+c \vec{u})=R
$$

(c denotes the concentration, $\mathrm{D}$ the diffusion coefficient, $\mathrm{z}$ the charge, $\mathrm{K}$ the ion mobility, $\mathrm{R}$ the chemical reaction rate of the ionic species of interest, $\mathrm{F}$ the Faraday constant, $\mathrm{V}$ the electrical potential, and $u$ the velocity field of the bulk fluid.) Details of the transport equation are found for example in [9]. The ion mobility is defined as the proportional factor between the constant migration velocity $v_{\mathrm{m}}$ of ions in an electrical field with the field strength $\mathrm{E}$ at viscous conditions [10]:

$$
v_{m}=K E
$$

Equation (1) may be combined with other models which describe the electrical field (i.e., Poisson's equation) and the bulk fluid flow (i.e., Navier-Stoke's equations). The combined equation system may be solved for specific boundary conditions with appropriate numerical methods.

Both models have been used to successfully describe the ion motion in different types of IMS $[11,12]$. However, to the best of our knowledge, there was no direct comparison between the numerical approaches with experimental results. Furthermore, we are not aware of any applications of the numerical methods for the simulation of ion motion within relative complex geometries and relatively fast gas flows. Such conditions drastically raise the complexity and costs of the modeling process.
In this paper, we present a thorough comparison of two approaches for the numerical simulation of ion motion in relatively fast gas flows at atmospheric pressure in the presence of an electric field directed orthogonally to the main gas flow direction: particle tracing calculations applying the SIMION program package, including the SDS user program and electro-kinetic flow simulations with the Comsol Multiphysics program package. Both models require flow dynamic simulations of the bulk gas motion as input parameters. The flow dynamic data sets were also computed with Comsol Multiphysics. We demonstrate the effects of various simulation parameters on the model performance and compare the calculated results with experimental data.

This paper is the second in a series on experimentally validated ion motion simulations and attempts to lay the foundations for the treatment of increasingly complex geometries, such as current commercially available AP ion sources. The first paper in the series described in detail the procedures required for computational flow dynamics (CFD) simulations within complex geometries [13]. The present paper examines the interplay between viscous and electrical forces on ions moving at atmospheric pressure. A third paper currently in preparation describes efforts to model ion motion within a standard API source, again with experimental validation of the simulation results [14].

\section{Experimental}

\section{Vacuum Chamber/Ion Current Measurement}

All experiments were performed using a home-built setup. In essence it consisted of a sealed stainless steel vacuum chamber (i.d.: $127 \mathrm{~mm}$, height: $131 \mathrm{~mm}$ ), which was equipped with an assembly of two electrodes, i.e., a deflection and a detection electrode. Figure 1 shows a

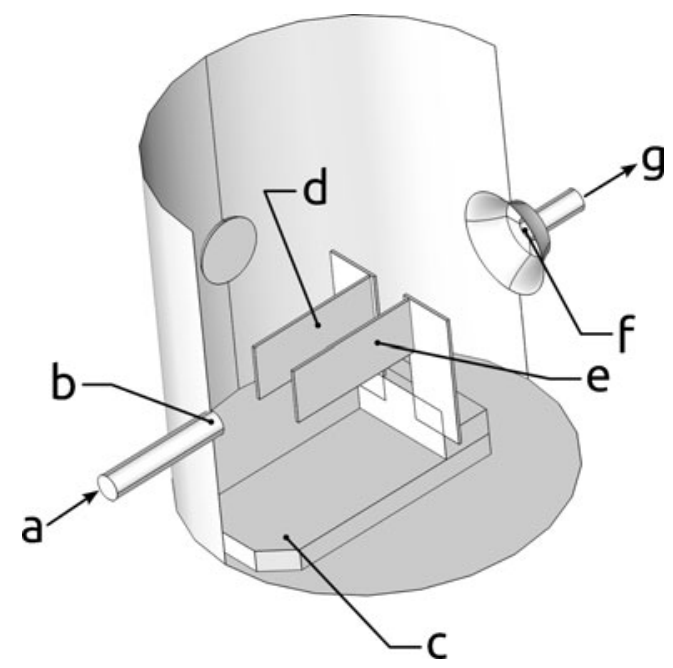

Figure 1. Schematic of the experimental setup. (a) Gas flow from ion source. (b) Inlet port. (c) Moveable electrode assembly. (d) Deflection electrode. (e) Detection electrode. (f) Outlet port. (g) Gas flow to rough pump 
schematic of the setup. The chamber was evacuated with a $2 \mathrm{~m}^{3} \mathrm{~h}^{-1}$ rough pump (Leybold Trivac model D2A; Oerlikon Leybold Vacuum GmbH, Cologne, Germany). The pumping speed was adjusted with a needle valve. The tubular ion source (i.d.: $9 \mathrm{~mm}$, length: $90 \mathrm{~mm}$ ) was coupled to the chamber on the far side of the pumping port via a connection tube (i.d.: $9 \mathrm{~mm}$, length: $90 \mathrm{~mm}$ ). The two rectangular electrodes (48 $\mathrm{mm}$ width, $20 \mathrm{~mm}$ height) were mounted on a movable isolated PVC assembly. The electrodes were aligned on the center axis between the chamber inlet and outlet port at a distance of $18 \mathrm{~mm}$ with the gas flow passing between them. The ion current from the detection electrode was measured with a Model $610 \mathrm{C}$ electrometer/microammeter (Keithley Instruments Inc., Cleveland, OH, USA). The voltage on the deflection electrode (up to 80 VDC) was provided by a laboratory power supply (Voltcraft PSP 1803; Conrad Electronic SE, Hirschau, Germany). Note that just the voltage on the deflection electrode is changed during the experiments. The voltage of the detection electrode, which is mounted face to face to the deflection electrode (cf. Figure 1) remains constant, i.e., is grounded via the ammeter input resistance. Vacuum feed-throughs were used for electrical connections. The total gas flow was controlled with a $2000 \mathrm{sccm} \mathrm{min}^{-1}$ mass flow controller (MKS Instruments, Andover, MA, USA) and the chamber gas pressure was measured with a Barocel 600A-1000T pressure transducer (Datametrics/Dresser, Wilmington, MA, USA) mounted on a side port.

\section{Ion Source}

Ions were generated with a corona discharge in a custom built tubular ion source. A medical injection needle $(0.6 \mathrm{~mm}$ diameter, $60 \mathrm{~mm}$ length; B. Braun Melsungen AG, Melsungen, Germany) was used as the point electrode and was placed on the center axis of a metal tube serving as grounded plate electrode. Dry Nitrogen gas of $99.999 \%$ purity (Gase.de Vertriebs GmbH, Sulzbach, Germany) was fed through the injection needle at atmospheric pressure; the chamber's effluent was vented into the exhaust system of the laboratory. The $2 \mathrm{kV}$ corona needle voltage was provided by a $\mathrm{HNC}$ 3500-10 ump power supply (Heinziger Electronic $\mathrm{GmbH}$, Rosenheim, Germany). Ions originating from the corona discharge were transported by laminar viscous flow into the chamber via the connection tube.

\section{Methods}

\section{Fluid Flow Simulation}

The finite element method (FEM) software package Comsol Multiphysics ver. 4.0 and ver. 4.0a (Comsol AB, Stockholm, Sweden), termed "Comsol" in the remainder of this paper, were used for the simulation of three-dimensional stationary computational fluid dynamics (CFD) models of the gas flow in the chamber. A weakly compressible formulation of the
Navier Stokes equations [15] was applied, which is a reasonable assumption for flow velocities with Mach numbers $<0.3$. Details about the mathematical formulation are available in the Comsol documentation manual [16]. An isothermal flow at a temperature of $298 \mathrm{~K}$ in nitrogen was assumed for all calculations; thus, the temperature distribution was not included in the simulation runs. The dynamical viscosity of the $\mathrm{N}_{2}$ gas at the given temperature was interpolated by the material property functions for $\mathrm{N}_{2}$ provided by Comsol. The actual value at $298 \mathrm{~K}$ was $17.6 \mu \mathrm{Pa} \mathrm{s}^{-1}$. The boundary conditions for the flow simulations were a static pressure of $p_{i n}=1$ bar at the inlet boundary and a fixed outflow velocity between 0.05 and $1.35 \mathrm{~ms}^{-1}$ at the outlet boundary. The pressure inside the chamber was initialized with $p_{\text {chamber }}=1$ bar. We considered chamber walls with friction leading to negligible local gas velocities ("no slip" condition). The deflection and receiver electrodes were modeled in subsequent simulations with and without friction ("slip" and "no slip" conditions), respectively.

\section{Ion Motion Simulations}

\section{Comsol Multiphysics}

The ion motion was modeled based on the fluid dynamical simulations described above using an electrostatic model with the "Electrostatics Module" of Comsol. For details of the mathematical background confer the Comsol documentation [16]. The boundary conditions for this model were an adjustable potential between 0 and $80 \mathrm{~V}$ for the deflection electrode and ground potential for all other boundaries including the detection electrode. The "Transport of Diluted Species" interface of the Chemical Reaction Engineering Module of Comsol Multiphysics was initialized with distinct ion mobilities ranging between 1.2 and $3.5 \times 10^{-4} \mathrm{~m}^{2} \mathrm{~V}^{-1} \mathrm{~s}^{-1}$, respectively, an average isotropic diffusion coefficient of $1 \times 10^{-5} \mathrm{~m}^{2} \mathrm{~s}^{-1}$, and an electrical charge of $\mathrm{z}=+1$. The chosen ion mobility range resulted from a given mapping algorithm between ion masses and ion mobility. For details about the mapping, see the SIMION/SDS simulations and the Discussion section. If not noted otherwise, a static inflow ion concentration of $\mathrm{c}_{\mathrm{in}}=6 \times 10^{11} \mathrm{~mol} \mathrm{~m}^{-3}$, corresponding to $3.6 \times$ $10^{7}$ molecule $\mathrm{cm}^{-3}$ with convective outflow at the outlet boundary was assumed. The inflow ion concentration was estimated from measured absolute ion current data. For all remaining walls ideal termination of the ionic species was assumed, leading to a fixed ion wall concentration of zero $\left(\mathrm{c}_{\mathrm{w}}=0\right)$.

In selected simulation runs, the effects of space charge were investigated by coupling the ion migration and the electrostatic model. For this purpose, the inflow ion concentration was multiplied by the Faraday constant and directly considered as a distributed space charge in the electrostatic model (mono polar space charge model). In subsets of the latter simulations, a second ionic species with identical properties but $\mathrm{z}=-1$ (bipolar space charge model) was included as well. 
Finally, the numerical surface integral of the ion concentration on the receiver electrode was interpreted as ion current signal.

\section{SIMION SDS Simulations}

Charged particle tracings were performed with SIMION ver. 8.0.4 along with the SDS implementation shipped with the software package. The results of the CFD simulations $(0.45$, $0.55,0.85$, and $1.35 \mathrm{~ms}^{-1}$ outflow velocity, respectively) were transferred to the SDS user program with a proprietary Matlab (Release 2010a; Mathworks Inc., Natick, MA, USA) export script. A simplified version of the geometry of the CFD model was replicated as a truly scaled SIMION potential array (PA).

The simulated particles had analogous properties to the ionic species in the Comsol model. In contrast to the Comsol model, though, the statistical particle tracing approach within SIMION allows the simulation of the motion of a distribution of ions with different masses and mobilities. We assumed a uniform ion mass range distribution between $\mathrm{m} / \mathrm{z}$ 19 and 350. The selected ion mobility range in the continuous model roughly corresponds to this mass range in the SIMION model. Details of the used mapping function between ion mass and ion mobility used in the SDS simulation algorithm are found in the work by Appelhans and Dahl [7]. As in the Comsol model, mixed sets of anions and cations were simulated, applying the same voltages to both electrodes and chamber walls, as described above.
Additionally, a set of simulations was run with an additional potential applied on the receiver electrode to investigate the effects of a (rather speculative) charged boundary layer on the electrode surface.

For virtual ion current determinations, ion traces terminating on the receiver electrode in the simulation run were counted as function of time intervals by means of a further custom Matlab script, which analyzed the "ion fly" record files generated by SIMION. The number of simulated ions per run was typically between 5000 and 10,000. As before, space charge effects such as ion repulsion were taken into account by activating the "Coulombic Repulsion" feature of SIMION in some of the simulation runs.

\section{Results and Discussion}

\section{Fluid Flow Simulations}

The stationary flow dynamical simulations obtained with the Comsol program package reveal a relatively simple flow structure inside the chamber, as expected. Figure 2 shows the simulation results obtained for two different gas velocities. Most of the injected gas flows through the chamber within a rather confined geometry. There is no significant velocity loss of the gas in the confined flow region with only a very subtle spreading visible. The disturbance of the flow by the electrode plates is negligible. There is, however, a noticeable feature at the outflow port of the geometry: Here the sharp edges of the outlet flange peels

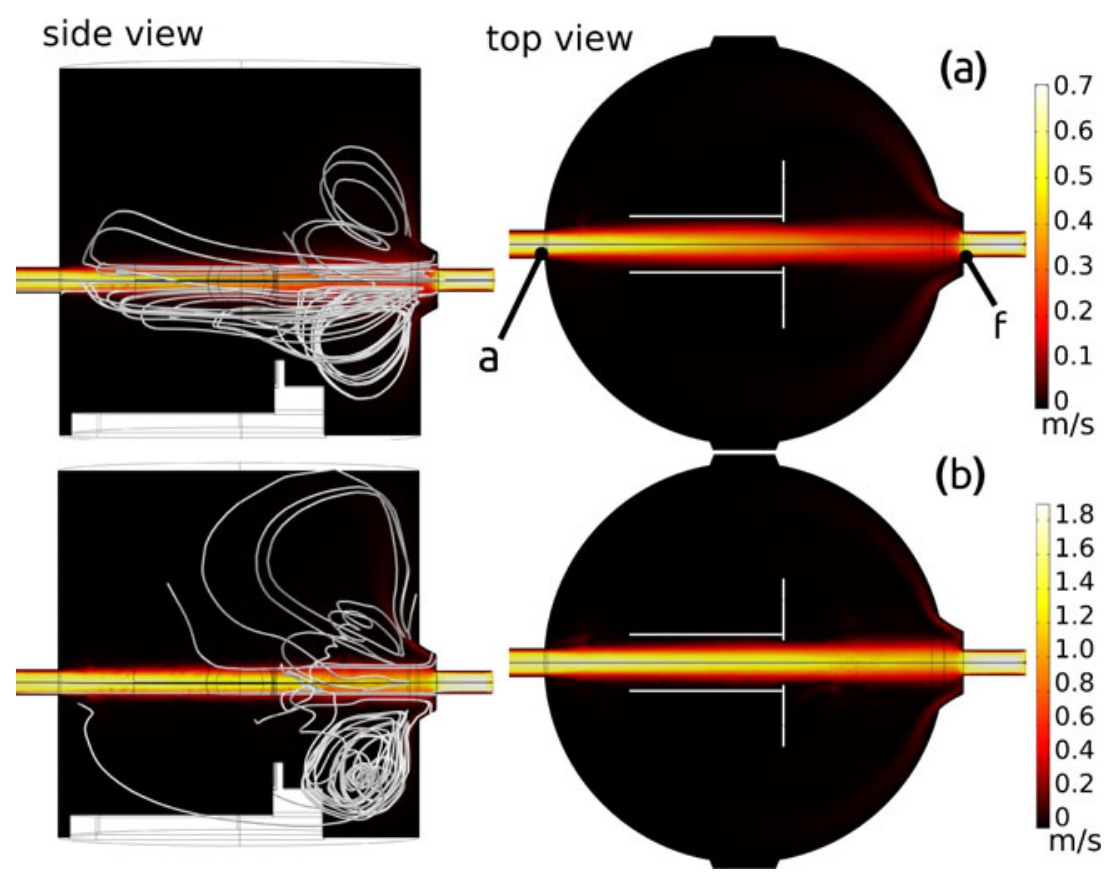

Figure 2. FEM simulations of a gas flow entering the chamber as shown in Figure 1 at 1 bar total pressure. Upper panels "a": Exit mean gas velocity $0.45 \mathrm{~ms}^{-1}$. Lower panels "b": Exit mean gas velocity $1.35 \mathrm{~ms}^{-1}$. The gas flow enters from the left port "a". Note the backflow reflected by the exit port "f" (cf. Figure 1) and the minor changes in the central flow structure when the gas flow velocity increases. The white flow lines on the left panels show the different structure of the flow in the bulk volume induced by the reflected part of the gas stream. The backflow does not interfere with the center flow 
off the outer layer of the slightly broadened gas stream and reflects it. This backflow adapts to the chamber walls and is clearly discernible in the velocity plots. However, the backflow dissipates into the bulk volume of the chamber (not approaching the detection electrode) and the gas velocities decrease rapidly leading to negligible interferences.

The flow lines on the left of Figure 2 indicate that the flow outside the main confined region is probably nonstationary and turbulent. The stationary solution derived by Comsol thus allows only a rough visualization of the flow structure in this area. For a more complete picture of the flow conditions in the bulk volume, time-dependent CFD calculations and turbulence modeling would be necessary. However, the flow outside the main flow region seems to have no significant effect on the main flow itself, thus the integrity of the results shown here is still given with rather high confidence levels. From a comparison of the results shown in Figure 2a and $\mathrm{b}$ follows that despite increasing gas velocities the shape of the expansion is not significantly affected. The general picture is the same for the backflow: the shape is only minimally altered with increased gas velocity; the backflow becomes sharper and more confined.

In summary, the flow simulations show that in the given velocity interval, increasing gas velocities do not severely change the general features of the flow structure. Comparisons of the results with and without friction on both electrode surfaces show only very subtle differences at the mean gas velocities simulated. Detailed analysis of the gas velocity distribution between the electrodes reveals that there is virtually no difference in this area, even at further elevated mean gas velocities (data not shown). This is basically caused by the relatively low gas velocities prevailing in the envelope region of the main gas stream. The diameter of the gas stream is obviously governed by the inlet port diameter; wider port diameters would inevitably lead to an intensive interaction between friction forces at the electrodes surface and the gas flow.

\section{Experimental Results}

The ion current originating from the Corona discharge was measured as function of the voltage on the deflection electrode at an inflow rate of $1.1 \mathrm{Lmin}^{-1}$. This volume flow corresponds to a mean inflow velocity of $0.28 \mathrm{~ms}^{-1}$.

As shown in Figure 3, the measured ion current reaches a maximum at a deflection voltage of approximately $15 \mathrm{~V}$. At lower and higher values, the ion current exhibits a nearly linear dependence on the deflection voltage, with a steep incline between 0 and $15 \mathrm{~V}$ and a far less pronounced almost linear decline at higher voltages. At $80 \mathrm{~V}$ deflection voltage, the ion current has almost dropped to its original value without deflection. This experimental data set is used as reference for the simulation results shown below.

Of note, the almost identical absolute ion current recorded with positive and negative deflection voltages at the deflection electrodes, cf. Figure 3, is remarkable. The corona discharge voltage was kept constant at $+2 \mathrm{kV}$ in both experiments. This suggests that the corona discharge in the ion source produced virtually identical concentrations of anions and cations and that both ionic polarities are transported from the needle region into the measurement chamber with comparable efficiencies. It seems to be highly unlikely that a disproportionate production of ions with positive and negative polarity would be quantitatively compensated by different transport efficiencies. This situation is rationalized in terms of fast electron conversion to form positive ions and efficient thermal electron capture by trace gases present in the $\mathrm{N}_{2}$ flow: the generally accepted corona plasma initiated ion production route [3] is briefly summarized below. The reaction sequences are far from being complete; they are intended to rationalize the selected range of ion masses for the simulations. It is safe to assume that with the simple experimental set-up used, oxygen and water are present in significant amounts in the ion source region reaching at least several hundred ppmV mixing ratios. Thus, a relatively broad range of clustered charge carriers is expected to be present in the gas flow [3]. The ion populations are readily thermodynamically equilibrated; collisionally induced dissociation reactions do not occur at atmospheric pressure with the voltages applied. For positive ions RXNs RXN1-RXN4 apply, for negative ions, RXNs RXN5-RXN7.

$$
\begin{array}{r}
\mathrm{N}_{2}+\mathrm{e}_{\text {(fast })} \rightarrow \mathrm{N}_{2}^{+}+2 \mathrm{e} \\
\mathrm{N}_{2}^{+}+\mathrm{H}_{2} \mathrm{O} \rightarrow \mathrm{N}_{2}+\mathrm{H}_{2} \mathrm{O}^{+} \\
\mathrm{H}_{2} \mathrm{O}^{+}+\mathrm{H}_{2} \mathrm{O} \rightarrow \mathrm{H}_{3} \mathrm{O}^{+}+\mathrm{OH}
\end{array}
$$

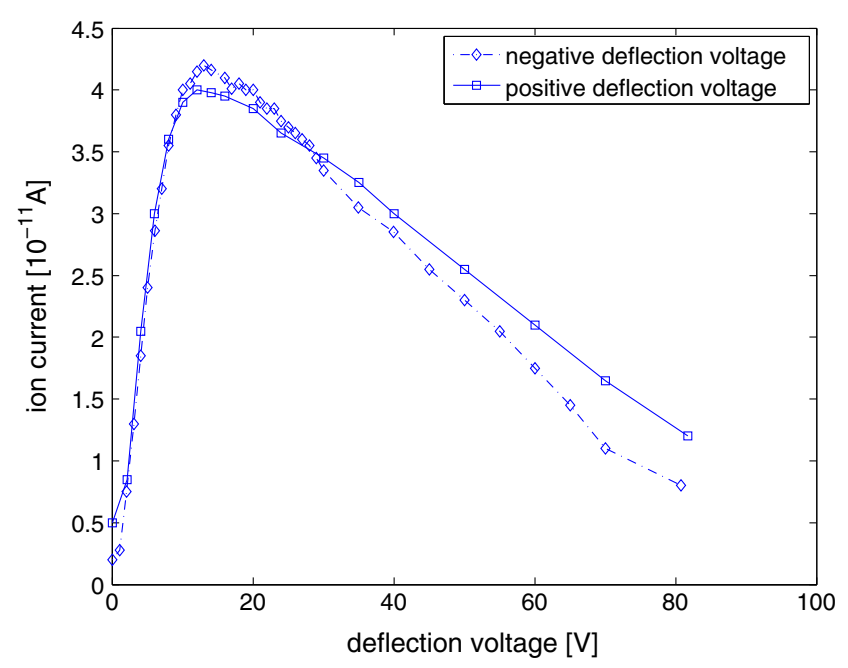

Figure 3. Experimentally determined ion current as function of deflection voltage. Note the virtually linear rise and fall off regions below $10 \mathrm{~V}$ and above $20 \mathrm{~V}$, respectively 


$$
\mathrm{H}_{3} \mathrm{O}^{+}+\mathrm{n} \mathrm{H}_{2} \mathrm{O} \rightarrow\left[\mathrm{H}+(\mathrm{n}+1) \mathrm{H}_{2} \mathrm{O}\right]^{+} ; \mathrm{n}=4,5,6, \ldots
$$

(RXN4)

$$
\begin{gathered}
\mathrm{e}+\mathrm{M} \rightarrow \mathrm{e}_{(\text {therm })}+\mathrm{M} \\
\mathrm{e}_{(\text {therm })}+\mathrm{O}_{2} \rightarrow \mathrm{O}_{2}{ }^{-} \\
\mathrm{O}_{2}{ }^{-}+\mathrm{n} \mathrm{H}_{2} \mathrm{O} \rightarrow\left[\mathrm{O}_{2}+\mathrm{n} \mathrm{H}_{2} \mathrm{O}\right]^{-} ; \mathrm{n}=4,5,6, \ldots
\end{gathered}
$$

(RXN7)

$\mathrm{M}$ denotes a non-energized bath gas species, $\mathrm{e}_{\text {(fast) }}$ electrons in the hot discharge region, and $\mathrm{e}_{(\text {therm })}$ collisionally cooled (thermalized) electrons. There are numerous subsequent reaction cascades such as the generation of clustered $\mathrm{OH}^{-}$ anions and many other species. It is emphasized that the initially extremely reactive species, e.g., $\mathrm{H}_{3} \mathrm{O}^{+}$and $\mathrm{O}_{2}^{-}$lose their reactivity almost entirely by clustering with water molecules. This leads to a corona effluent in which positively and negatively charged clustered ions do not undergo extensive neutralization reactions, as observed in the experiments.

\section{SIMION/SDS Simulations}

Figure 4 shows a set of results obtained with the ion trajectory simulations using SIMION/SDS. As expected, at low deflection voltages ions are transported past the measurement electrode by the viscous gas flow (cf. $0 \mathrm{~V}$ deflection voltage plot in Figure 4). With increasing deflection voltage, the ions are directed towards the detection electrode (cf. $10 \mathrm{~V}$ deflection voltage plot in Figure 4). With further increasing voltage on the deflection electrode, the electrical forces exerted on the ions exceed the viscous forces. As a result, the ions are pushed against the chamber walls and, thus, entirely miss the receiver electrode (cf. $80 \mathrm{~V}$ deflection voltage plot in Figure 4). These results very nicely match qualitatively the experimentally found ion current signals which were recorded as function of the deflection voltage, cf. Figure 3.

A more quantitative comparison is shown in Figure 5, left panel. Here, simulated ion currents on the detection electrode computed with the SIMION/SDS model are shown, again as function of deflection electrode potential, and for three different gas flows. The ion mobility was assumed to be equally distributed between $1.0 \times 10^{-4}$ and $3.6 \times 10^{-4} \mathrm{~m}^{2} \mathrm{~V}^{-1} \mathrm{~s}^{-1}$, corresponding to a (calculated) homogeneous mass distribution between $\mathrm{m} / \mathrm{z} 18$ and 350 . In each run, the computed ion response is in very good qualitative agreement with the experimentally observed response curve. The simulated ion currents reproduce the linear steep signal incline and less pronounced linear fall off very nicely. In addition, the maxima of the simulated ion current shift with increasing gas flow, as observed in the experiments. As expected, the maximum signal intensity is shifted towards higher deflection potentials with rising bulk gas velocities because the viscous drag forces on the ions increase. Obviously the ions penetrate deeper into the deflection field region at elevated viscous drag forces.

A quantitative comparison reveals that the numerical model data match with the experimentally determined data when assuming a mean gas velocity which is roughly 2.8 times higher than the experimental value (cf. Figure 5, left panel). Errors in the measured gas flow and chamber pressure cannot account for this difference. It is noted though, that gas velocity and ion mobility have opposite effects on the computed ion signal. This behavior is illustrated in Figure 5, right panel, which shows a comparison of impact of the average gas velocity and the ion mobility on the computed ion current. It becomes readily apparent that decreasing ion mobility and increasing gas velocity have more or less an inversely proportional effect on the numerically observed ion current with the SIMION/SDS model.

This finding suggests that the ion mobility distribution assumed in the simulations may not correctly reflect the ion population present in the experiment, i.e., the mean of the mobility distribution may be up-to a factor of 3 too high in the simulation. Simulations with single ion mobilities or much narrower mobility distributions revealed that there is no severe impact of the selected width on the general shape of the simulated ion current response. The results are

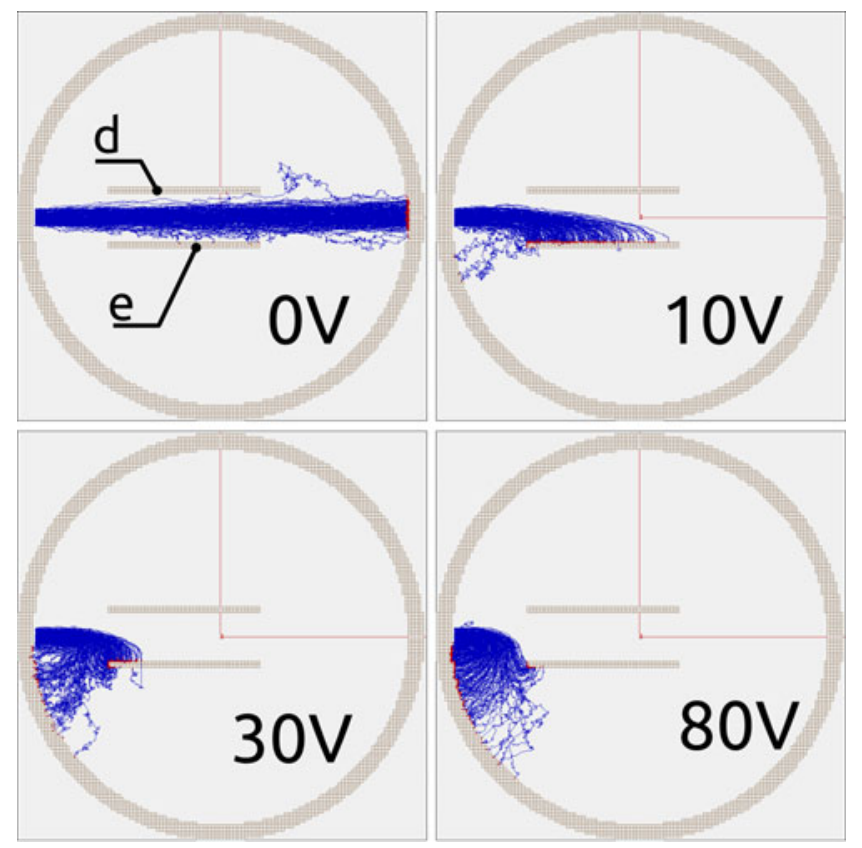

Figure 4. Simulated ion trajectories using the SIMION/SDS approach. The FEM flow simulation "a" in Figure 2 was used as input data set. Deflection voltages are given in the Figure. The labels on the electrodes ("d", "e") are identical to the labels in Figure 1, i.e., the plate labeled with " $\mathrm{e}$ " is the detection electrode 

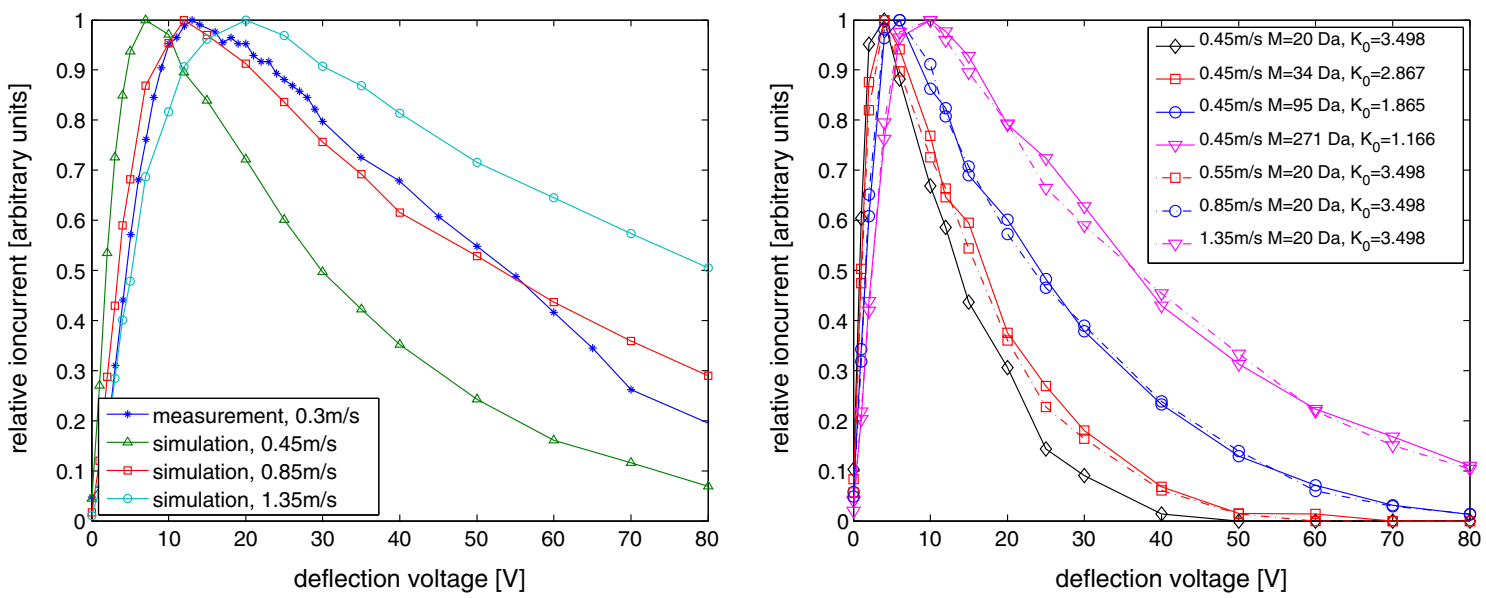

Figure 5. Comparison of calculated ion current on receiver electrode in SIMION/SDS simulations with experimental data. Left panel: Equally distributed ion mobilities between 3.6 and $1.0 \times 10^{-4} \mathrm{~m}^{2} \mathrm{~V}^{-1} \mathrm{~s}^{-1}$. According to the mobility-mass mapping procedure provided in SIMION, this corresponds to a mass range of $m / z$ 18-350. Right panel: Comparison of the impact of the gas velocity and ion mobility on the calculated ion current in SIMION/SDS simulations. The reduced ion mobility $\mathrm{K}_{0}$ is given in units of $10^{-4} \mathrm{~m}^{2} \mathrm{~V}^{-1} \mathrm{~s}^{-1}$

comparable to those shown for varying mean flow speeds or the mean value of the ion mobility.

Other causes for the difference between simulation and experiment could either be space charge effects or noticeable charging of the measurement electrode due to buildup of a charged layer on the metal surface of the electrode. The SIMION/SDS approach is not capable of simulating the interaction between space charge and the electrical field distribution. It is possible, though, to activate a Coulombic repulsion model, which simulates the electrostatic repulsion between the charged particles. Simulation runs with activated Coulombic repulsion showed only insignificant differences to the results obtained without a repulsion model activated. It is noted though that space charge effects cannot be entirely excluded as cause for the difference between the SIMION/ SDS simulation and measurement. Particularly, space charge shielding of the electrical field between deflection and detection electrode could potentially account for the difference between simulation and experiment. A highly resistive layer on the metal surface could lead to a noticeable electrical potential on the receiver electrode. To investigate the effects of such a charge buildup, we conducted a set of simulations with an additional potential applied to the receiver electrode. This potential was adjusted proportional to the ion current, which was determined experimentally for a given deflection voltage. In this case, the simulated ion current deviates even further from the experimental results and thus significant charge build-up is not considered further.

In conclusion, an overestimated mean ion mobility appears to be most likely responsible for the difference between SIMION / SDS simulation and experimental results.

\section{Comsol Ion Migration Simulations}

One example of a simulation result of the ion migration model using Comsol is shown in Figure 6. The calculated ion concentration distributions are entirely consistent with the simulation results obtained with SIMION/SDS. As illustrated before, the ions are noticeably pushed towards the detection electrode at relatively small deflection voltages. The ion current reaches a maximum when most of the ions impinge on the detection electrode (labeled $10 \mathrm{~V}$ in Figure 6). At higher voltages on the deflection electrode, the electrical forces exceed the viscous forces and transport of the ions into the volume between the electrodes is suppressed. Instead, the ions are directed towards the chamber walls and the ion current signal drops.

A remarkable feature in the ion migration simulation results is a zone of high ion concentration on the receiver electrode surface, clearly discernible in Figure $6(10 \mathrm{~V}$ deflection voltage). Most probably, this is caused by the low gas velocities in close proximity to the receiver electrode. Initially, the ions are pushed towards the receiver electrode into this zone of low gas velocity where viscous drag forces strongly decrease. Thus a "cushion" of high ion concentration is building up.

The ion current signals obtained with the Comsol ion migration model (Figure 7) shows roughly the same features as the SIMION/SDS simulation results. Increasing gas velocities and decreasing ion mobilities shifts the maximum of the numerical ion current signal to higher deflection voltages and the initial current rise is significantly steeper than the following decline.

A significant difference to the SIMION/SDS results is the pronounced curvature of the declining signal, which is not observed in the experiments. Furthermore, in contrast to the simulations results obtained with SIMION/SDS, the mean gas velocity and the ion mobility are not exactly inversely affecting the simulated ion current signal. This is demonstrated in Figure 7. Obviously, both parameters have rather different effects over almost the entire deflection voltage range. Only at deflection voltages exceeding $50 \mathrm{~V}$ (Figure 7, left) the signals begin to converge. The right panel of 

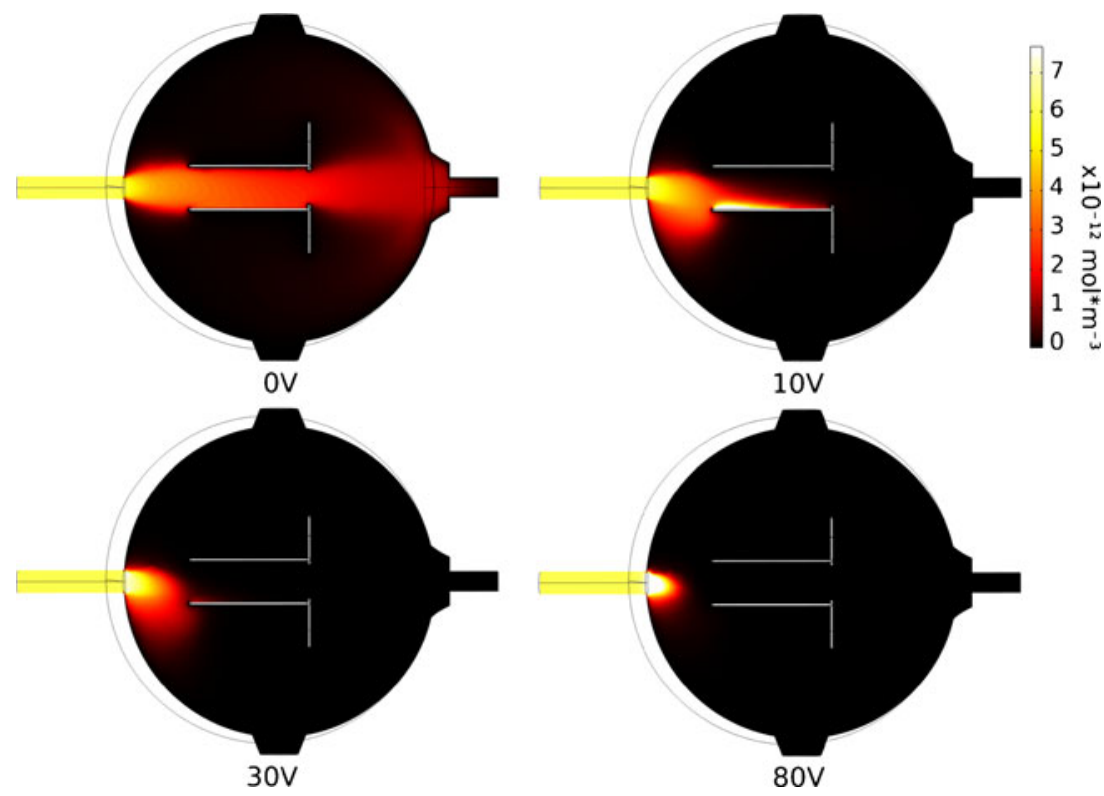

Figure 6. Ion concentration distribution plots obtained with the Comsol ion migration model. The ion mobility is $3.0 \times 10^{-4} \mathrm{~m}^{2}$ $\mathrm{V}^{-1} \mathrm{~s}^{-1}$ and the mean gas outflow velocity is $0.45 \mathrm{~ms}^{-1}$ (the flow simulation "a" in Figure 2 is used)

Figure 7 furthermore shows that the position of the ion signal maximum differs significantly from experimental data.

Nevertheless it is possible to "force" a reasonably good agreement between the simulated ion current and the experimental data (cf. Figure 8), however, with considerably larger differences in the parameter values gas velocity and ion mobility, respectively. A satisfactory "match" does not occur until the flow speed is assumed to be $1.35 \mathrm{~ms}^{-1}$. Furthermore, relatively low ion mobility values corresponding to ion masses well above $270 \mathrm{Da}$, according to the mobility mass mapping function provided by the SDS algorithm [7], are required. This seems to be rather unlikely; preliminary mass resolved measurements of the ion popula-

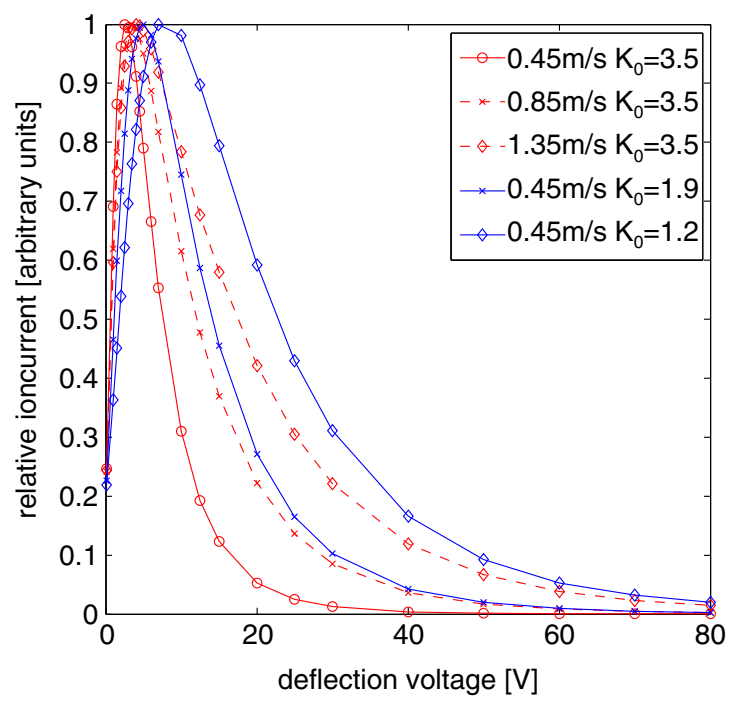

tion generated in the presently used corona discharge source suggest a distribution maximum around $\mathrm{m} / \mathrm{z} 100$ and 150 . This matches with literature data, where the $\left[\mathrm{H}+\mathrm{nH}_{2} \mathrm{O}\right]^{+}$ and $\left[\mathrm{O}_{2}+\mathrm{nH}_{2} \mathrm{O}\right]^{-}$clusters have a distribution maximum at $n=5,6$ under typical corona conditions. In addition to the results of the SIMION simulations, this finding supports the notion that the used mobility-mass mapping may not predict the actual ion mobilities correctly under the experimental conditions. For future work, the application of scattering models [17], which allow obtaining the ion mobilities from geometric parameters of ions, could provide a much better base for realistic ion mobility range estimations.

It is worth mentioning that the inability to run simulations assuming a distribution of ion mobilities is a fundamental

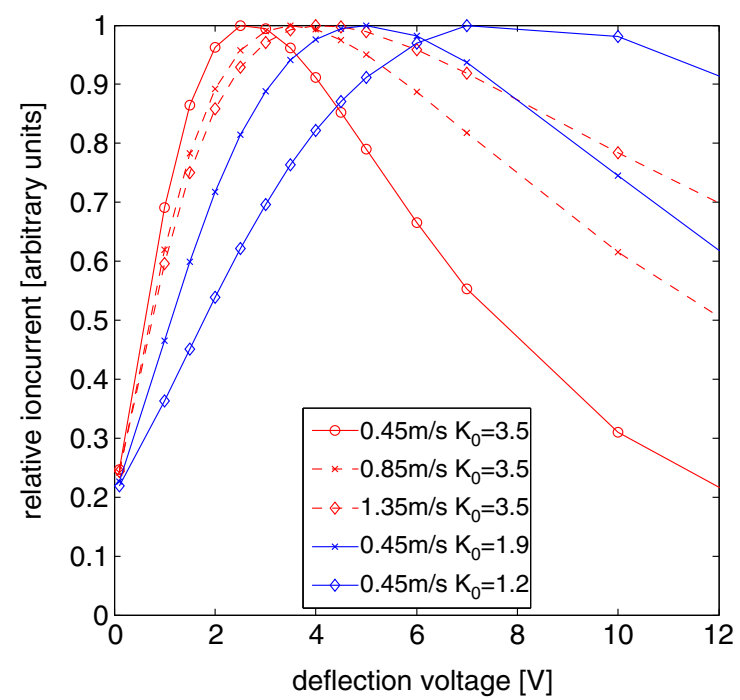

Figure 7. Comparison of the impact of the gas velocity and ion mobility on the calculated ion current in Comsol simulations. The reduced ion mobility $\mathrm{K}_{0}$ is given in units of $10^{-4} \mathrm{~m}^{2} \mathrm{~V}^{-1} \mathrm{~s}^{-1}$ 


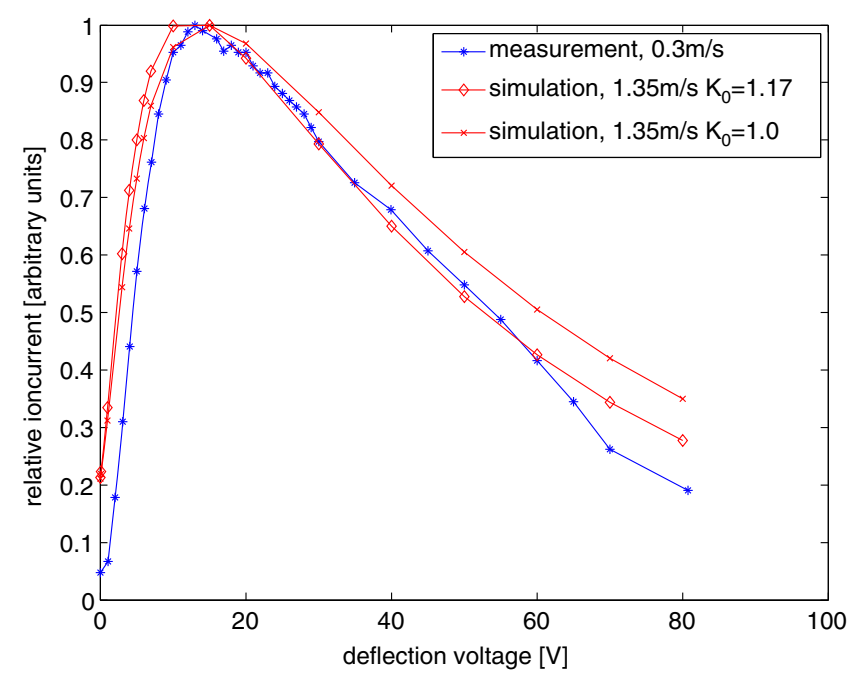

Figure 8. Comparison of calculated ion current on receiver electrode in Comsol simulations with experimental data. For the simulations, a reduced ion mobility of $10^{-4} \mathrm{~m}^{2} \mathrm{~V}^{-1} \mathrm{~s}^{-1}$ was assumed

weakness of the ion migration model in Comsol. To accurately model the characteristics of the - probably rather wide-ion mobility distribution produced by a corona discharge ion source, the Comsol model calls for the introduction of a characteristic set of ionic species. This approach causes a significant impact on the Comsol migration model, particularly with respect to numerical costs and numerical stability (see below).

Comsol simulations with deactivated friction on the two electrodes ("slip" boundary condition) show no significant impact on the computed ion current signals, particularly the "cushion" of high ion concentrations remained stable, as expected. As stated earlier, the low gas velocities in this region were almost independent from the slip condition on the electrode surfaces, thus the viscous forces on the ions are also not affected. The consideration of the space charge in a mono- and bipolar space charge model had barely noticeable effects on the simulation results.

\section{Numerical Stability and Cost}

The ion concentration is computed in the Comsol model using partial differential equations, which are solved with a finite element method process. In contrast to the direct particle tracing approach in SIMION/SDS, the FEM solver easily becomes numerically unstable and reaches no convergence. In the calculations for this paper, numerical stability was an issue, particularly with respect to the fluid flow model. By activating the space charge simulation, the electrical and the ion migration model becomes closely coupled, potentially affecting the stability of the entire simulation. In terms of numerical costs, a comparable picture arises. The fluid flow model is by far the most complex and, thus, the most expensive model. In terms of absolute computing times, the ion migration models cost more, since the simulation required much more stationary ion migration solutions than fluid flow solutions.

It should be noted that the consideration of space charge significantly raises the numerical costs for both simulation approaches. In particular, for the SIMION/ SDS model, activated space charge (i.e., Coulombic repulsion) limited the number of simulated particles per simulation run to approximately 2000. With higher particle numbers, the simulation process becomes unreasonably slow, which may adversely affect the level of statistical confidence.

\section{Summary and Conclusion}

The computational results shown in this paper demonstrate that even for relatively complex three-dimensional geometries, fluid flow, ion migration, and ion trajectory simulations, including the consideration of space charge, are successfully modeled on advanced consumer class computer hardware.

Both the ion migration model in Comsol and the SIMION/SDS model are qualitatively reproducing the experimentally recorded ion currents. A comparison of both approaches shows that the setup of the Comsol model is much more difficult, tends to become numerically unstable, and the computed results were in relatively poor agreement with the experimental results. In particular the numerical stability issues and the definition of adequate boundary conditions was a significant drawback for the ion migration model. A further investigation of the cause of the observed differences between experiment and the numerical models would require the validation of the assumed ion mobility distribution and the validation of the whole fluid flow model, which represented the basis for both ion motion models. This is not the scope of the current paper and will be addressed in more depth in upcoming contributions.

It is remarkable that without detailed knowledge of the ion mobility distribution produced by the corona discharge ion source along with the simplified gas flow model for the experimental chamber, the SIMION/SDS results are only differing roughly by a factor of 3 from the experimental results. In this regard the question arises whether or not the Comsol model is necessary at all, considering that the SIMION/SDS model setup is much easier, is numerically stable, and produces better results. For stationary flow conditions the answer is probably "no." There are at least two issues affecting the applicability of the SIMION/SDS approach though: (1) in many real world cases, the fluid flow is non stationary, i.e., may change rapidly with time. There is currently no way to consider such conditions in SIMION/SDS. Thus, for non-stationary cases, reliable ion trajectory simulations with SIMION/SDS will only be possible with severe simplifications, if at all. (2) SIMION/ SDS provides only very limited space charge corrections, in particular the interaction between space charge and external electrical fields is not taken into account. 
In contrast, Comsol is basically designed to handle both issues raised above but demands a much higher investment in the model definition. Furthermore, the Comsol model results represent a rather abstract continuous ion concentration distribution. In most cases, the individual ion trajectories, including the calculation of individual ion transport times, are much more illustrative in the perception of the results. The possibility of directly interacting with individual particles in user programs is perhaps the most important advantage of the SIMSION/SDS approach. Generally, the complex boundary conditions are much easier to define in terms of direct instructions for individual particles rather than in terms of abstract partial differential calculus.

In summary, both models showed individual advantages and drawbacks. For relatively simple, stationary cases SIMION/SDS with validated CFD input is considered the better choice. In case of complex multi physics couplings, e.g., ion generation from evaporating droplets within a hot gas jet or the evolution of ion populations originating from a corona discharge needle with high space charge present and additional ion generation by the inevitable presence of vacuum ultraviolet light, complex FEM models as provided by Comsol and similar systems are unavoidable. In many cases, the numerical costs would not be a limiting factor for the solution of a modeling problem if advanced computer hardware is available, but the costs caused by the correct initialization, adequate problem definition, and the necessary validation of the models, in particular of the CFD model, certainly will.

\section{Acknowledgments}

The authors acknowledge funding in part for this work by the German Research Foundation (DFG), contracts BE 2124/6-1 and BE 2124/4-1. The authors thank the reviewers of this manuscript for their helpful comments and suggestions.

\section{References}

1. Scientific Instrument Services Inc.: SIMION ver. 8.0, Ringoes, NJ, USA

2. Bruins, A.P., Cook, K.D: Electrospray ionization: principles and instrumentation. van Berkel, G.J.: Electrochemistry of the electrospray ionization source; Covey, T.R., Kovarik, P., Jong, R: Pneumatically assisted electrospray ionization. In: The Encyclopedia of Mass Spectrometry, Vol. 6, Ionization Methods, 1st ed., Gross, M.L., Caprioli, R.N. (eds.) Elsevier, Oxford, U.K. (2007)

3. Moini, M.: Atmospheric pressure chemical ionization: principles, instrumentation, and applications. In: Gross, M.L., Caprioli, R.N. (eds.) The Encyclopedia of Mass Spectrometry, Vol. 6, Ionization Methods, 1st edn. Elsevier, Oxford (2007)

4. Mukhopadhyay, R.: IMS/MS: its time has come. Anal. Chem. 80, 7918-7920 (2008)

5. Valentine, S.J., Kurulugama, R.T., Bohrer, B.C., Merenbloom, S.I., Sowell, R.A., Mechref, Y., Clemmer, D.E.: Developing IMS-IMS-MS for rapid characterization of abundant proteins in human plasma. Int. J. Mass Spectrom. 283, 149-160 (2009)

6. Sone, Y.: Molecular Gas Dynamics, 8th edn. Birkhäuser, Boston (2007)

7. Appelhans, A.D., Dahl, D.A.: SIMION ion optics simulations at atmospheric pressure. Int. J. Mass Spectrom. 244, 1-14 (2005)

8. Appelhans, A.D., Dahl, D.A.: Measurement of external ion injection and trapping efficiency in the ion trap mass spectrometer and comparison with a predictive model. Int. J. Mass Spectrom. 216, 269284 (2002)

9. Comsol, A.B.: Chemical Reaction Engineering Module User's Guide ver. 4.0a, 1998-2010

10. Eiceman, G.A., Karpas, Z.: Ion Mobility Spectrometry, 2nd edn. CRC Press, Boca Raton (2005)

11. Lai, H., McJunkin, T.R., Miller, C.J., Scott, J.R., Almirall, J.R.: The predictive power of SIMION/SDS simulation software for modeling ion mobility spectrometry instruments. Int. J. Mass Spectrom. 276, 1-8 (2008)

12. Barth, S., Zimmermann S.: Modeling Ion Motion in a Miniaturized Ion Mobility Spectrometer. Proceedings of the European COMSOL Conference, Hannover, Germany (2008)

13. Poehler, T., Kunte, R., Hoenen, H., Jeschke, P., Wissdorf, W., Brockmann, K.J., Benter, T.: Numerical simulation and experimental validation of the three-dimensional flow field and relative analyte concentration distribution in an atmospheric pressure ion source. J. Am. Soc. Mass Spectrom. (2011). doi:10.1007/s13361-011-0211-z

14. Wissdorf, W., Lorenz, M., Klee, S., Poehler, T., Hoehnen, H., Benter, T.: Atmospheric pressure ion source development: experimental validation of simulated ion trajectories within complex flow velocity and electrical fields. Unpublished (manuscript in preparation)

15. Ferzinger, J.H., Perić, M.: Computational Methods for Fluid Dynamics, 3rd edn. Springer, Berlin (2002)

16. Comsol, A.B.: Comsol Multiphysics ver. 4.0a Users Guide, 1998-2010

17. Shvartsburg, A.A., Jarrold, M.F.: An exact hard-spheres scattering model for the mobilities of polyatomic ions. Chem. Phys. Lett. 261, 8691 (1996) 